\title{
Ruthenium complexes of chelating amido-functionalized N-heterocyclic carbene ligands: Synthesis, structure and DFT studies
}

\author{
SACHIN KUMAR ${ }^{\mathrm{a}}$ ANANTHA NARAYANAN ${ }^{\mathrm{a}}$, MITTA NAGESWAR RAO ${ }^{\mathrm{a}}$, \\ MOBIN M SHAIKH ${ }^{\mathrm{b}}$ and PRASENJIT GHOSH ${ }^{\mathrm{a}, *}$ \\ ${ }^{a}$ Department of Chemistry and ${ }^{\mathrm{b}}$ National Single Crystal X-ray Diffraction Facility, Indian Institute of \\ Technology Bombay, Powai, Mumbai 400076, India \\ e-mail:pghosh@chem.iitb.ac.in
}

\begin{abstract}
Synthesis, structure and density functional theory (DFT) studies of a series of new ruthenium complexes, [1-(R)-3- $N$-(benzylacetamido)imidazol-2-ylidene]RuCl( $p$-cymene) $\left[\mathrm{R}=\mathrm{Me}(\mathbf{1 c}), i\right.$ - $\mathrm{Pr}(\mathbf{2 c}), \mathrm{CH}_{2} \mathrm{Ph}$ $(3 \mathrm{c}) ; p$-cymene $=4$ - $i$-propyltoluene] supported over $N / O$-functionalized $N$-heterocyclic carbene (NHC) ligands are reported. In particular, the ruthenium (1-3)c complexes were synthesized from the respective silver complexes, [1-(R)-3- $N$-(benzylacetamido)imidazol-2-ylidene ${ }_{2} \mathrm{Ag}^{+} \mathrm{Cl}^{-}\left[\mathrm{R}=\mathrm{Me}(\mathbf{1 b}), i-\mathrm{Pr}(\mathbf{2 b}), \mathrm{CH}_{2} \mathrm{Ph}(\mathbf{3 b})\right]$ by the treatment with $\left[\mathrm{Ru}(p \text {-cymene }) \mathrm{Cl}_{2}\right]_{2}$ in $65-76 \%$ yields. The molecular structures of $(\mathbf{1 - 3}) \mathbf{c}$ revealed the chelation of the N-heterocylic carbene ligand through the carbene center and an amido sidearm of the ligand in all of the three complexes. The density functional theory studies on the ruthenium (1-3)c complexes indicated strong binding of the NHC ligand to the metal center as was observed from the deeply buried NHC-Ru $\sigma$-bonding molecular orbitals.
\end{abstract}

Keywords. Carbenes; ruthenium; DFT; amido-functionalized NHC.

\section{Introduction}

Ruthenium adds several new and interesting options to the ever-growing world of transition metal mediated organic methodologies, in constant demand for meeting the everyday challenges of organic synthesis. ${ }^{1}$ For instance, the ruthenium complexes display selective $\mathrm{C}-\mathrm{H}$ activation reactions of particular relevance to synthesizing biaryl and heterocyclic frameworks, ${ }^{2}$ and thereby providing useful alternatives to the already overstretched palladium mediated carboncarbon cross-coupling reactions. ${ }^{3}$ As such, the $\mathrm{C}-\mathrm{H}$ activations, and more so the selective $\mathrm{C}-\mathrm{H}$ activation ones, fall among the difficult but much desired challenges, currently confronting the world of synthesis today. ${ }^{4}$ Apart from the elegant selective $\mathrm{C}-\mathrm{H}$ activation reactions, the ruthenium engages in a variety of other synthetically important transformations namely, asymmetric transfer hydrogenation reactions, ${ }^{5}$ metathesis, ${ }^{6}$ olefin epoxidation reactions, ${ }^{7}$ hydroformylation reactions, ${ }^{8}$ electrooxidation reactions, ${ }^{9}$ facial cycloaddition of azides with terminal alkynes regioselectively ${ }^{10}$ etc. to name a few.

The phenomenal success of the N-heterocyclic carbenes in homogeneous catalysis, has also made them

\footnotetext{
${ }^{*}$ For correspondence
}

increasingly popular as ligands for stabilizing transition metal complexes. ${ }^{11}$ Easy tunablity of the sterics and the electronic environments through constant variation of the substituents on the nitrogen atom of the imidazole ring, make these ligands versatile in stabilizing a variety of transition metal complexes in varied oxidation states. With our interests being in biomedical ${ }^{12}$ and catalytic applications ${ }^{13}$ of transition metal complexes of N-heterocyclic carbene ligands, ${ }^{14}$ we became involved in synthesizing its ruthenium complexes for their potential utility in chemical catalysis.

In this contribution, we report a series of ruthenium complexes, [1-(R)-3- $N$-(benzylacetamido)imidazol-2ylidene] $\mathrm{RuCl}(p$-cymene) $[\mathrm{R}=\mathrm{Me}(\mathbf{1 c}), i-\operatorname{Pr}(\mathbf{2 c})$, $\mathrm{CH}_{2} \mathrm{Ph}(3 \mathbf{3}) ; p$-cymene $=4-i$-propyltoluene], supported over $\mathrm{N} / \mathrm{O}$-functionalized $\mathrm{N}$-heterocyclic carbene ligands (figure 1). The Ru-NHC interaction in the (13)c complexes have been investigated using density functional theory (DFT) studies.

\section{Experimental}

\subsection{General procedures}

All manipulations were carried out using a combination of a glove box and standard Schlenk techniques. 


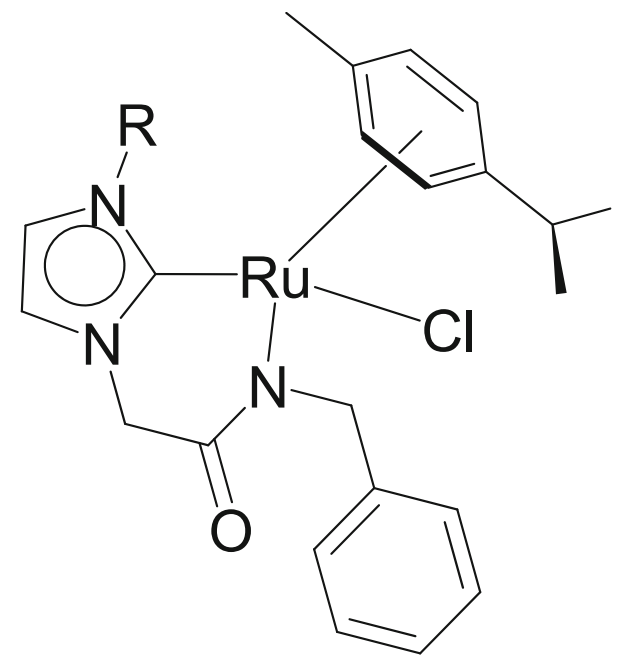

$\mathrm{R}=\mathrm{Me}(\mathbf{1 c}), i-\operatorname{Pr}(\mathbf{2 c}), \mathrm{CH}_{2} \mathrm{Ph}(\mathbf{3 c})$

Figure 1. Ruthenium (1-3)c complexes of chelating amido-functionalized N-heterocyclic carbene ligands.

Solvents were purified and degassed by standard procedures. $\mathrm{RuCl}_{3} \bullet \mathrm{xH}_{2} \mathrm{O}$ was purchased from $\mathrm{SD}$-fine Chemicals (India) and used without any further purification. The 1a, ${ }^{15}(\mathbf{2}-\mathbf{3}) \mathbf{b}^{16}$ and $\left[\mathrm{Ru}(p \text {-cymene }) \mathrm{Cl}_{2}\right]_{2}{ }^{17}$ were synthesized according to reported literature procedures. ${ }^{1} \mathrm{H}$ and ${ }^{13} \mathrm{C}\left\{{ }^{1} \mathrm{H}\right\}$ NMR spectra were recorded on a Varian $400 \mathrm{MHz}$ NMR spectrometer. ${ }^{1} \mathrm{H}$ NMR peaks are labelled as singlet (s), doublet (d), triplet (t), and septet (sept). Infrared spectra were recorded on a Perkin Elmer Spectrum One FT-IR spectrometer. Mass spectrometry measurements were done on a Micromass Q-Tof spectrometer. The electronic spectra were recorded in acetonitrile by using Jasco V-570 UV/VIS/NIR Spectrophotometer. Cyclic voltammetric measurements were carried out using a BAS Epsilon electrochemistry system. Elemental Analyses were carried out on Thermo Quest FLASH 1112 SERIES (CHNS) Elemental Analyzer. X-ray diffraction data for (1-3)c were collected on an Oxford Diffraction Excaliber-S diffractometer and crystal data collection and refinement parameters are summarized in Supporting Information table S1. The structures were solved using direct methods and standard difference map techniques, and were refined by full-matrix least-squares procedures on $F^{2}$ with SHELXTL (Version 6.10). ${ }^{18}$

\subsection{Synthesis of $\{[1-($ methyl)-3- $N$-(benzylacetamido) imidazol-2-ylidene $]_{2} \mathrm{Ag}^{+} \mathrm{Cl}^{-}(\mathbf{1 b})$}

A mixture of 1-(methyl)-3-N-(benzylacetamido) imidazolium chloride (1a) $(4.37 \mathrm{~g}, 16.4 \mathrm{mmol})$ and
$\mathrm{Ag}_{2} \mathrm{O}$ (2.02 g, $8.72 \mathrm{mmol}$ ) in dichloromethane ( $c a$. $40 \mathrm{~mL}$ ) was stirred at room temperature for $4 \mathrm{~h}$. The reaction mixture was then filtered and the solvent was removed under vacuum to give the product $\mathbf{1 b}$ as off-white solid $(2.31 \mathrm{~g}, 47 \%) .{ }^{1} \mathrm{H}$ NMR $\left(\mathrm{CDCl}_{3}\right.$, $\left.400 \mathrm{MHz}, 25^{\circ} \mathrm{C}\right): \delta 9.36(\mathrm{br}, 1 \mathrm{H}, \mathrm{N} \underline{H}), 7.36(\mathrm{br}$, $1 \mathrm{H}, \mathrm{NC} \underline{H} \mathrm{CHN}), 7.29$ (br, $\left.2 \mathrm{H}, \mathrm{C}_{6} \underline{H}_{5}\right), 7.11(\mathrm{~m}, 3 \mathrm{H}$, $\left.\mathrm{C}_{6} \underline{H}_{5}\right), 6.94(\mathrm{br}, 1 \mathrm{H}, \mathrm{NCHC} \underline{H \mathrm{~N}}), 5.29\left(\mathrm{br}, \mathrm{C}_{2}\right)$, 4.36 (s, 2H, $\left.\mathrm{C} \underline{H}_{2}\right), 3.77$ (s, $\left.3 \mathrm{H}, \mathrm{C} \underline{H}_{3}\right) \cdot{ }^{13} \mathrm{C}\left\{{ }^{1} \mathrm{H}\right\} \mathrm{NMR}$ $\left(\mathrm{CDCl}_{3}, 100 \mathrm{MHz}, 25^{\circ} \mathrm{C}\right): \delta 182.3(\mathrm{~N} \underline{\mathrm{CN}}-\mathrm{Ag}), 167.2$ ( $\underline{C O}), 138.5$ (ipso- $\left.\underline{C}_{6} \mathrm{H}_{5}\right), 128.4\left(m-\underline{C}_{6} \mathrm{H}_{5}\right), 128.1$ $\left(o-\underline{C}_{6} \mathrm{H}_{5}\right), 126.9\left(p-\underline{C}_{6} \mathrm{H}_{5}\right), 123.4(\mathrm{~N} \underline{\mathrm{CHCHN}}), 121.6$ $(\mathrm{NCH} \underline{C} \mathrm{HN}), 53.9 \quad\left(\mathrm{CO} \underline{\mathrm{C}} \mathrm{H}_{2}\right), 43.2\left(\underline{\mathrm{C}} \mathrm{H}_{2} \mathrm{NH}\right), 38.8$ $\left(\mathrm{CH}_{3}\right)$. IR (KBr Pellet): $1676\left(v_{\mathrm{CO}}\right) \mathrm{cm}^{-1}$. Anal. calcd. for $\mathrm{C}_{26} \mathrm{H}_{30} \mathrm{AgN}_{6} \mathrm{O}_{2} \mathrm{Cl} \bullet \mathrm{CH}_{2} \mathrm{Cl}_{2}: \mathrm{C}, 47.22 ; \mathrm{H}, 4.70 ; \mathrm{N}$, 12.24. Found: C, 47.46; H, 5.03, N, 11.84 .

\subsection{Synthesis of [1-(methyl)-3-N-(benzylacetamido) imidazol-2-ylidene]Ru(p-cymene) $\mathrm{Cl}$ (1c)}

A mixture of $\{[1-($ methyl)-3- $\{\mathrm{N}$-(benzylacetamido) imidazol-2-ylidene $\left.\}]_{2} \mathrm{Ag}\right\}^{+} \mathrm{Cl}^{-}(\mathbf{1 b})(0.302 \mathrm{~g}, 0.502 \mathrm{mmol})$ and $\left[\mathrm{Ru}(p \text {-cymene }) \mathrm{Cl}_{2}\right]_{2} \quad(0.307 \mathrm{~g}, 0.502 \mathrm{mmol})$ was dissolved in dichloromethane $(c a .40 \mathrm{~mL})$ and was stirred at room temperature for $6 \mathrm{~h}$, when the formation of an off-white $\mathrm{AgCl}$ precipitate was observed. The reaction mixture was then filtered and the solvent was removed under vacuum to yield the crude product as a brown solid. The crude product 1c was purified by column chromatography on a silica gel by elution with a $\mathrm{CHCl}_{3} / \mathrm{CH}_{3} \mathrm{OH}(\mathrm{v} / \mathrm{v}$ 10:1) mixed medium to give the product $1 \mathrm{c}$ as brown solid $(0.181 \mathrm{~g}, 72 \%) .{ }^{1} \mathrm{H}$ NMR $\left(\mathrm{CDCl}_{3}, 400 \mathrm{MHz}, 25^{\circ} \mathrm{C}\right): \delta 7.50\left(\mathrm{~d}, 2 \mathrm{H},{ }^{3} \mathrm{~J}_{\mathrm{HH}}=\right.$ $\left.8 \mathrm{~Hz}, o-\mathrm{C}_{6} \underline{H}_{5}\right), 7.35\left(\mathrm{~m}, 3 \mathrm{H}, m / p-\mathrm{C}_{6} \underline{H}_{5}\right), 7.00(\mathrm{~d}$, $\left.1 \mathrm{H},{ }^{3} \mathrm{~J}_{\mathrm{HH}}=2 \mathrm{~Hz}, \mathrm{NC} \underline{H} \mathrm{CHN}\right), 6.97\left(\mathrm{~d}, 1 \mathrm{H},{ }^{3} \mathrm{~J}_{\mathrm{HH}}=\right.$ $2 \mathrm{~Hz}, \mathrm{NCHC} \underline{H} \mathrm{~N}), 5.31\left(\mathrm{~d}, 1 \mathrm{H},{ }^{2} \mathrm{~J}_{\mathrm{HH}}=15 \mathrm{~Hz}, \mathrm{C} \underline{H}_{2}\right)$, $5.13\left(\mathrm{~d}, 1 \mathrm{H},{ }^{3} \mathrm{~J}_{\mathrm{HH}}=6 \mathrm{~Hz}, p-\mathrm{CH}_{3} \mathrm{C}_{6} \underline{H}_{4} \mathrm{CH}\left(\mathrm{CH}_{3}\right)_{2}\right)$, $4.89\left(\mathrm{~d}, 1 \mathrm{H},{ }^{3} \mathrm{~J}_{\mathrm{HH}}=6 \mathrm{~Hz}, p-\mathrm{CH}_{3} \mathrm{C}_{6} \underline{H}_{4} \mathrm{CH}\left(\mathrm{CH}_{3}\right)_{2}\right)$, $4.83\left(\mathrm{~d}, 1 \mathrm{H},{ }^{3} \mathrm{~J}_{\mathrm{HH}}=6 \mathrm{~Hz}, p-\mathrm{CH}_{3} \mathrm{C}_{6} \underline{H}_{4} \mathrm{CH}\left(\mathrm{CH}_{3}\right)_{2}\right)$, $4.82\left(\mathrm{~d}, 1 \mathrm{H},{ }^{3} \mathrm{~J}_{\mathrm{HH}}=6 \mathrm{~Hz}, p-\mathrm{CH}_{3} \mathrm{C}_{6} \underline{H}_{4} \mathrm{CH}\left(\mathrm{CH}_{3}\right)_{2}\right)$, $4.46\left(\mathrm{~s}, 2 \mathrm{H}, \mathrm{C}_{H_{2}}\right), 4.45\left(\mathrm{~d}, 1 \mathrm{H},{ }^{2} \mathrm{~J}_{\mathrm{HH}}=15 \mathrm{~Hz}\right.$, $\left.\mathrm{C} \underline{H}_{2}\right), 3.99$ (s, $\left.3 \mathrm{H}, C \underline{H}_{3}\right), 2.45$ (sept, $1 \mathrm{H},{ }^{3} \mathrm{~J}_{\mathrm{HH}}=$ $\left.7 \mathrm{~Hz}, \quad p-\mathrm{CH}_{3} \mathrm{C}_{6} \mathrm{H}_{4} \mathrm{C} \underline{H}\left(\mathrm{CH}_{3}\right)_{2}\right), \quad 1.98 \quad(\mathrm{~s}, \quad 3 \mathrm{H}, \quad p-$ $\left.\mathrm{C}_{3} \mathrm{C}_{6} \mathrm{H}_{4} \mathrm{CH}\left(\mathrm{CH}_{3}\right)_{2}\right), 1.11\left(\mathrm{~d}, 3 \mathrm{H},{ }^{3} \mathrm{~J}_{\mathrm{HH}}=7 \mathrm{~Hz}\right.$, $\left.p-\mathrm{CH}_{3} \mathrm{C}_{6} \mathrm{H}_{4} \mathrm{CH}\left(\mathrm{C}_{3}\right)_{2}\right), 0.88\left(\mathrm{~d}, 3 \mathrm{H},{ }^{3} \mathrm{~J}_{\mathrm{HH}}=7 \mathrm{~Hz}\right.$, $\left.p-\mathrm{CH}_{3} \mathrm{C}_{6} \mathrm{H}_{4} \mathrm{CH}\left(\mathrm{C}_{\underline{H}_{3}}\right)_{2}\right) .{ }^{13} \mathrm{C}\left\{{ }^{1} \mathrm{H}\right\} \quad \mathrm{NMR} \quad\left(\mathrm{CDCl}_{3}\right.$, $\left.100 \mathrm{MHz}, 25^{\circ} \mathrm{C}\right): \delta 175.2(\mathrm{~N} \underline{\mathrm{CN}}-\mathrm{Ru}), 169.7(\underline{\mathrm{CO}})$, $143.6 \quad\left(\right.$ ipso- $\left.\underline{C}_{6} \mathrm{H}_{5}\right), \quad 128.7 \quad\left(m-\underline{C}_{6} \mathrm{H}_{5}\right), \quad 128.0 \quad(o-$ $\left.\underline{C}_{6} \mathrm{H}_{5}\right), 126.1\left(p-\underline{C}_{6} \mathrm{H}_{5}\right), 122.8(\mathrm{~N} \underline{C H C H N}), 122.0$ $(\mathrm{NCH} C \mathrm{HN}), \quad 107.4$ (ipso- $\left.p-\mathrm{CH}_{3} \bar{C}_{6} \mathrm{H}_{4} \mathrm{CH}\left(\mathrm{CH}_{3}\right)_{2}\right)$, 100.1 (ipso- $\left.p-\mathrm{CH}_{3} \mathrm{C}_{6} \mathrm{H}_{4} \mathrm{CH}\left(\mathrm{CH}_{3}\right)_{2}\right), \quad 88.6 \quad$ ( $p$ $\left.\mathrm{CH}_{3} \underline{C}_{6} \mathrm{H}_{4} \mathrm{CH}\left(\mathrm{CH}_{3}\right)_{2}\right)$, 85.2 $\left(p-\mathrm{CH}_{3} \underline{C}_{6} \mathrm{H}_{4} \mathrm{CH}\left(\mathrm{CH}_{3}\right)_{2}\right)$, 
$83.0 \quad\left(p-\mathrm{CH}_{3} \mathrm{C}_{6} \mathrm{H}_{4} \mathrm{CH}\left(\mathrm{CH}_{3}\right)_{2}\right), \quad 81.9 \quad(p-$ $\left.\mathrm{CH}_{3} \underline{C}_{6} \mathrm{H}_{4} \mathrm{CH}\left(\mathrm{CH}_{3}\right)_{2}\right), 55.5\left(\mathrm{CO}_{\underline{C}} \mathrm{H}_{2}\right), 55.4\left(\underline{\mathrm{C}} \mathrm{H}_{2} \mathrm{NH}\right)$, $37.8\left({ }_{C} \mathrm{H}_{3}\right), 31.5\left(p-\mathrm{CH}_{3} \mathrm{C}_{6} \mathrm{H}_{4} \underline{C} \mathrm{C}\left(\mathrm{CH}_{3}\right)_{2}\right), 24.9(p-$ $\left.\mathrm{CH}_{3} \mathrm{C}_{6} \mathrm{H}_{4} \mathrm{CH}\left(\underline{\mathrm{C}} \mathrm{H}_{3}\right)_{2}\right), \quad 20.1 \quad\left(p-\mathrm{CH}_{3} \mathrm{C}_{6} \mathrm{H}_{4} \mathrm{CH}\left(\underline{\mathrm{C}} \mathrm{H}_{3}\right)_{2}\right)$, $18.7\left(p-\underline{C} \mathrm{H}_{3} \mathrm{C}_{6} \mathrm{H}_{4} \mathrm{CH}\left(\mathrm{CH}_{3}\right)_{2}\right)$. IR (KBr Pellet): 1686 $\left(v_{\mathrm{CO}}\right) \mathrm{cm}^{-1}$. Anal. calcd. for $\mathrm{C}_{23} \mathrm{H}_{28} \mathrm{RuN}_{3} \mathrm{OCl}$ : C, 55.36; H, 5.66; N, 8.42. Found: C, 55.35; H, 6.02; N, 8.34 .

2.4 Synthesis of [1-(i-propyl)-3-N-(benzylacetamido) imidazol-2-ylidene ]Ru(p-cymene) Cl (2c)

A mixture of $\{[1-(i$-propyl)-3- $\{\mathrm{N}$-(benzylacetamido) imidazol-2-ylidene $\left.\}]_{2} \mathrm{Ag}\right\}^{+} \mathrm{Cl}^{-}$(2b) $(0.331 \mathrm{~g}, 0.505 \mathrm{mmol})$ and $\left[\mathrm{Ru}(p \text {-cymene }) \mathrm{Cl}_{2}\right]_{2}(0.308 \mathrm{~g}, 0.505 \mathrm{mmol})$ was dissolved in dichloromethane (ca. $40 \mathrm{~mL}$ ) and was stirred at room temperature for $6 \mathrm{~h}$, when the formation of an off-white $\mathrm{AgCl}$ precipitate was observed. The reaction mixture was then filtered and the solvent was removed under vacuum to yield the product $\mathbf{2 c}$ as a brown solid. The crude product $\mathbf{2 c}$ was purified by column chromatography on a silica gel by elution with a $\mathrm{CHCl}_{3} / \mathrm{CH}_{3} \mathrm{OH}(\mathrm{v} / \mathrm{v}$ 10:1) mixed medium to give the product $2 \mathbf{c}$ as brown solid $(0.202 \mathrm{~g}, 76 \%) .{ }^{1} \mathrm{H}$ $\operatorname{NMR}\left(\mathrm{CDCl}_{3}, 400 \mathrm{MHz}, 25^{\circ} \mathrm{C}\right): \delta 7.47\left(\mathrm{~d}, 2 \mathrm{H},{ }^{3} \mathrm{~J}_{\mathrm{HH}}=\right.$ $\left.7 \mathrm{~Hz}, o-\mathrm{C}_{6} \underline{H}_{5}\right), 7.32\left(\mathrm{br}, 2 \mathrm{H}, m-\mathrm{C}_{6} \underline{H}_{5}\right), 7.29(\mathrm{br}, 1 \mathrm{H}$, $\mathrm{NC} \underline{H C H N}), 7.21\left(\mathrm{t}, 1 \mathrm{H},{ }^{3} \mathrm{~J}_{\mathrm{HH}}=7 \mathrm{~Hz}, p-\mathrm{C}_{6} \underline{H}_{5}\right), 6.95$ $(\mathrm{s}, \overline{1 \mathrm{H}}, \mathrm{NCHC} \underline{H} \mathrm{~N}), 5.23\left(\mathrm{~d}, 1 \mathrm{H},{ }^{2} \mathrm{~J}_{\mathrm{HH}}=15 \mathrm{~Hz}, \underline{\mathrm{C}}_{2}\right)$, $5.10\left(\mathrm{~d}, 1 \mathrm{H},{ }^{3} \mathrm{~J}_{\mathrm{HH}}=6 \mathrm{~Hz}, p-\mathrm{CH}_{3} \mathrm{C}_{6} \underline{H}_{4} \mathrm{CH}\left(\mathrm{CH}_{3}\right)_{2}\right)$, $5.10\left(\right.$ sept, $\left.1 \mathrm{H},{ }^{3} \mathrm{~J}_{\mathrm{HH}}=7 \mathrm{~Hz}, \mathrm{CH}\left(\mathrm{CH}_{3}\right)_{2}\right), 4.84(\mathrm{~d}, 1 \mathrm{H}$, $\left.{ }^{3} \mathrm{~J}_{\mathrm{HH}}=6 \mathrm{~Hz}, p-\mathrm{CH}_{3} \mathrm{C}_{6} \mathrm{H}_{4} \mathrm{CH}\left(\mathrm{CH}_{3}\right)_{2}\right), 4.81(\mathrm{~d}, 1 \mathrm{H}$, $\left.{ }^{3} \mathrm{~J}_{\mathrm{HH}}=6 \mathrm{~Hz}, p-\mathrm{CH}_{3} \mathrm{C}_{6} \underline{H}_{4} \mathrm{CH}\left(\mathrm{CH}_{3}\right)_{2}\right), 4.70(\mathrm{~d}, 1 \mathrm{H}$, $\left.{ }^{3} \mathrm{~J}_{\mathrm{HH}}=6 \mathrm{~Hz}, p-\mathrm{CH}_{3} \mathrm{C}_{6} \underline{H}_{4} \mathrm{CH}\left(\mathrm{CH}_{3}\right)_{2}\right), 4.43(\mathrm{~s}, 2 \mathrm{H}$, $\left.\mathrm{C} \underline{H}_{2}\right), 4.37\left(\mathrm{~d}, 1 \mathrm{H},{ }^{2} \mathrm{~J}_{\mathrm{HH}}=15 \mathrm{~Hz}, \mathrm{C} \underline{H}_{2}\right), 2.45$ (sept, $\left.1 \mathrm{H},{ }^{3} \mathrm{~J}_{\mathrm{HH}}=6 \mathrm{~Hz}, p-\mathrm{CH}_{3} \mathrm{C}_{6} \mathrm{H}_{4} \mathrm{C} \underline{H}\left(\mathrm{CH}_{3}\right)_{2}\right) 1.97(\mathrm{~s}, 3 \mathrm{H}$, $\left.p-\underline{\mathrm{C}}_{3} \mathrm{C}_{6} \mathrm{H}_{4} \mathrm{CH}\left(\mathrm{CH}_{3}\right)_{2}\right), 1.55\left(\mathrm{~d}, 3 \mathrm{H},{ }^{3} \mathrm{~J}_{\mathrm{HH}}=7 \mathrm{~Hz}\right.$, $\left.\mathrm{CH}\left(\underline{\mathrm{C}}_{3}\right)_{2}\right), 1.50\left(\mathrm{~d}, 3 \mathrm{H},{ }^{3} \mathrm{~J}_{\mathrm{HH}}=7 \mathrm{~Hz}, \mathrm{CH}\left(\mathrm{C}_{3}\right)_{2}\right)$, $1.11\left(\mathrm{~d}, 3 \mathrm{H},{ }^{3} \mathrm{~J}_{\mathrm{HH}}=7 \mathrm{~Hz}, p-\mathrm{CH}_{3} \mathrm{C}_{6} \mathrm{H}_{4} \mathrm{CH}\left(\mathrm{C}_{3}\right)_{2}\right), 0.86$ $\left(\mathrm{d}, 3 \mathrm{H},{ }^{3} \mathrm{~J}_{\mathrm{HH}}=7 \mathrm{~Hz}, p-\mathrm{CH}_{3} \mathrm{C}_{6} \mathrm{H}_{4} \mathrm{CH}\left(\mathrm{C}_{\mathrm{H}_{3}}\right)_{2}\right) .{ }^{13} \mathrm{C}\left\{{ }^{1} \mathrm{H}\right\}$ NMR $\left(\mathrm{CDCl}_{3}, 100 \mathrm{MHz}, 25^{\circ} \mathrm{C}\right): \delta 175.4(\mathrm{NCN}-\mathrm{Ru})$, 169.9 ( $\underline{C O}), 143.8\left(\right.$ ipso- $\left.\underline{C}_{6} \mathrm{H}_{5}\right), 128.8\left(m-\underline{C}_{6} \mathrm{H}_{5}\right), 128.2$ $\left(o-\underline{C}_{6} \mathrm{H}_{5}\right), 126.3\left(p-\underline{C}_{6} \mathrm{H}_{5}\right), 122.9(\mathrm{~N} \underline{C} \mathrm{HCHN}), 122.2$ $(\mathrm{NCH} \underline{C} \mathrm{HN}), \quad 107.6$ (ipso- $p-\mathrm{CH}_{3} \underline{C}_{6} \mathrm{H}_{4} \mathrm{CH}\left(\mathrm{CH}_{3}\right)_{2}$ ), 100.3 (ipso- $\left.p-\mathrm{CH}_{3} \mathrm{C}_{6} \mathrm{H}_{4} \mathrm{CH}\left(\mathrm{CH}_{3}\right)_{2}\right), \quad 88.8 \quad$ (p$\left.\mathrm{CH}_{3} \underline{C}_{6} \mathrm{H}_{4} \mathrm{CH}\left(\mathrm{CH}_{3}\right)_{2}\right), \quad 85.4\left(p-\mathrm{CH}_{3} \underline{C}_{6} \mathrm{H}_{4} \mathrm{CH}\left(\mathrm{CH}_{3}\right)_{2}\right)$, $83.2 \quad\left(p-\mathrm{CH}_{3} \underline{C}_{6} \mathrm{H}_{4} \mathrm{CH}\left(\mathrm{CH}_{3}\right)_{2}\right), \quad 82.1 \quad(p-$ $\left.\mathrm{CH}_{3} \underline{C}_{6} \mathrm{H}_{4} \mathrm{CH}\left(\mathrm{CH}_{3}\right)_{2}\right), 56.7\left(\mathrm{CO}^{\mathrm{C}} \mathrm{H}_{2}\right), 55.6\left(\underline{\mathrm{C}} \mathrm{H}_{2} \mathrm{NH}\right)$, $52.8 \quad\left(\underline{\mathrm{C}} \mathrm{H}\left(\mathrm{CH}_{3}\right)_{2}\right), \quad 52.5 \quad\left(\underline{\mathrm{C}} \mathrm{H}\left(\mathrm{CH}_{3}\right)_{2}\right), \quad 31.6 \quad(p-$ $\left.\mathrm{CH}_{3} \mathrm{C}_{6} \mathrm{H}_{4} \underline{\mathrm{C}} \mathrm{H}\left(\mathrm{CH}_{3}\right)_{2}\right)$, $25.1 \quad\left(p-\mathrm{CH}_{3} \mathrm{C}_{6} \mathrm{H}_{4} \mathrm{CH}\left(\underline{\mathrm{C}} \mathrm{H}_{3}\right)_{2}\right)$, $23.4 \quad\left(\mathrm{CH}\left(\mathrm{CH}_{3}\right)_{2}\right), \quad 23.2 \quad\left(\mathrm{CH}\left(\underline{\mathrm{CH}} \mathrm{H}_{3}\right)_{2}\right), \quad 20.3 \quad(p-$ $\left.\underline{\mathrm{C}} \mathrm{H}_{3} \mathrm{C}_{6} \mathrm{H}_{4} \mathrm{CH}\left(\mathrm{CH}_{3}\right)_{2}\right)$. IR (KBr Pellet): $1686\left(v_{C O}\right)$ $\mathrm{cm}^{-1}$. Anal. calcd. for $\mathrm{C}_{25} \mathrm{H}_{32} \mathrm{RuN}_{3} \mathrm{OCl} \bullet 0.5 \mathrm{CH}_{2} \mathrm{Cl}_{2}$ : C, 53.78; H, 5.84; N, 7.38. Found: C, 53.54; H, 6.23; N, 6.87.

\subsection{Synthesis of [1-(benzyl)-3-N-(benzylacetamido) imidazol-2-ylidene]Ru(p-cymene) $\mathrm{Cl}$ (3c)}

A mixture of \{[1-(benzyl)-3-\{N-(benzylacetamido) imidazol-2-ylidene $\left.\}]_{2} \mathrm{Ag}\right\}^{+} \mathrm{Cl}^{-}$(3b) $(0.421 \mathrm{~g}, 0.557 \mathrm{mmol})$ and $\left[\mathrm{Ru}(p \text {-cymene }) \mathrm{Cl}_{2}\right]_{2}(0.170 \mathrm{~g}, 0.278 \mathrm{mmol})$ was dissolved in dichloromethane $(c a .40 \mathrm{~mL})$ and was stirred at room temperature for $6 \mathrm{~h}$, when the formation of an off-white $\mathrm{AgCl}$ precipitate was observed. The reaction mixture was then filtered and the solvent was removed under vacuum to yield the crude product as a brown solid. The crude product 3c was purified by column chromatography on a silica gel by elution with a $\mathrm{CHCl}_{3} / \mathrm{CH}_{3} \mathrm{OH}$ (v/v 10:1) mixed medium to give the product $\mathbf{3 c}$ as brown solid $(0.208 \mathrm{~g}, 65 \%) .{ }^{1} \mathrm{H}$ NMR $\left(\mathrm{CDCl}_{3}, 400 \mathrm{MHz}, 25^{\circ} \mathrm{C}\right)$ : $\delta 7.50\left(\mathrm{br}, 1 \mathrm{H}, \mathrm{C}_{6} \underline{H}_{5}\right), 7.48\left(\mathrm{br}, 1 \mathrm{H}, \mathrm{C}_{6} \underline{H}_{5}\right), 7.41-$ 7.39 (br, 2H, $\mathrm{C}_{6} \underline{H}_{5}$ ), 7.36-7.34 (br, 3H, $\mathrm{C}_{6} \underline{H}_{5}$ ), 7.32 (br, $2 \mathrm{H}, \mathrm{C}_{6} \underline{H}_{5}$ ), 7.29 (br, $1 \mathrm{H}, \mathrm{C}_{6} \underline{H}_{5}$ ), 7.03 (br, $1 \mathrm{H}, \mathrm{NC} \underline{H C H N}), 6.91(\mathrm{br}, 1 \mathrm{H}, \mathrm{NCHC} \underline{H} \mathrm{~N}), 5.67$ $\left(\mathrm{d}, 1 \mathrm{H},{ }^{2} \mathrm{~J}_{\mathrm{HH}}=16 \mathrm{~Hz}, \mathrm{C} \underline{H}_{2}\right), 5.55\left(\mathrm{~d}, \overline{\mathrm{H}},{ }^{2} \mathrm{~J}_{\mathrm{HH}}=\right.$ $\left.16 \mathrm{~Hz}, \underline{\mathrm{H}}_{2}\right), 5.29\left(\mathrm{~d}, 1 \mathrm{H},{ }^{3} \mathrm{~J}_{\mathrm{HH}}=16 \mathrm{~Hz}, \underline{\mathrm{C}}_{2}\right), 4.91$ $\left(\mathrm{d}, 1 \mathrm{H},{ }^{3} \mathrm{~J}_{\mathrm{HH}}=6 \mathrm{~Hz}, \quad p-\mathrm{CH}_{3} \mathrm{C}_{6} \underline{H}_{4} \mathrm{CH}\left(\mathrm{CH}_{3}\right)_{2}\right), 4.75$ $\left(\mathrm{d}, 1 \mathrm{H},{ }^{3} \mathrm{~J}_{\mathrm{HH}}=6 \mathrm{~Hz}, p-\mathrm{CH}_{3} \mathrm{C}_{6} \underline{H}_{4} \mathrm{CH}\left(\mathrm{CH}_{3}\right)_{2}\right), 4.67$ $\left(\mathrm{d}, 1 \mathrm{H},{ }^{3} \mathrm{~J}_{\mathrm{HH}}=6 \mathrm{~Hz}, p-\mathrm{CH}_{3} \mathrm{C}_{6} \underline{H}_{4} \mathrm{CH}\left(\mathrm{CH}_{3}\right)_{2}\right), 4.48$ (s, 2H, $\mathrm{C}_{2}$ ), 4.44 (br, $1 \mathrm{H}, p-\mathrm{CH}_{3} \mathrm{C}_{6} \underline{H}_{4} \mathrm{CH}\left(\mathrm{CH}_{3}\right)_{2}$ ), $4.33\left(\mathrm{~d}, 1 \mathrm{H},{ }^{3} \mathrm{~J}_{\mathrm{HH}}=16 \mathrm{~Hz}, \mathrm{CH}_{2}\right), 2.46$ (sept, $\left.1 \mathrm{H},{ }^{3} \mathrm{~J}_{\mathrm{HH}}=6 \mathrm{~Hz}, \quad p-\mathrm{CH}_{3} \mathrm{C}_{6} \mathrm{H}_{4} \mathrm{CH}\left(\mathrm{CH}_{3}\right)_{2}\right), \quad 1.98$ (s, $\left.3 \mathrm{H}, \quad p-\mathrm{C}_{3} \mathrm{C}_{6} \mathrm{H}_{4} \mathrm{CH}\left(\mathrm{CH}_{3}\right)_{2}\right), \quad 1.11 \quad(\mathrm{~d}, \quad 3 \mathrm{H}$, $\left.{ }^{3} \mathrm{~J}_{\mathrm{HH}}=6 \mathrm{~Hz}, p-\mathrm{CH}_{3} \mathrm{C}_{6} \mathrm{H}_{4} \mathrm{CH}\left(\mathrm{CH}_{3}\right)_{2}\right), 0.88(\mathrm{~d}, 3 \mathrm{H}$, $\left.{ }^{3} \mathrm{~J}_{\mathrm{HH}}=6 \mathrm{~Hz}, \quad p-\mathrm{CH}_{3} \mathrm{C}_{6} \mathrm{H}_{4} \mathrm{CH}\left(\mathrm{C}_{3}\right)_{2}\right) .{ }^{13} \mathrm{C}\left\{{ }^{1} \mathrm{H}\right\}$ NMR $\left(\mathrm{CDCl}_{3}, 100 \mathrm{MHz}, 25^{\circ} \mathrm{C}\right): \delta 175.9(\mathrm{NCN}-$ $\mathrm{Ru}), \quad 169.8 \quad(\underline{C O}), \quad 143.0 \quad\left(\right.$ ipso- $\left.\underline{C}_{6} \mathrm{H}_{5}\right), \quad 136.90$

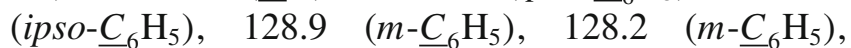
$128.1 \quad\left(o-\underline{C}_{6} \mathrm{H}_{5}\right), \quad 128.0 \quad\left(o-\underline{C}_{6} \mathrm{H}_{5}\right), \quad 127.9 \quad(p-$ $\left.\underline{C}_{6} \mathrm{H}_{5}\right), 127.1\left(p-\underline{C}_{6} \mathrm{H}_{5}\right), 122.1(\mathrm{~N} \underline{\mathrm{C}} \mathrm{HCHN}), 121.9$ $(\mathrm{NCH} \underline{C} \mathrm{HN}), \quad 107.8$ (ipso- $\left.p-\mathrm{CH}_{3} \underline{C}_{6} \mathrm{H}_{4} \mathrm{CH}\left(\mathrm{CH}_{3}\right)_{2}\right)$, 99.4 (ipso- $\left.p-\mathrm{CH}_{3} \mathrm{C}_{6} \mathrm{H}_{4} \mathrm{CH}\left(\mathrm{CH}_{3}\right)_{2}\right), \quad 87.6 \quad$ ( $p$ $\left.\mathrm{CH}_{3} \underline{C}_{6} \mathrm{H}_{4} \mathrm{CH}\left(\mathrm{CH}_{3}\right)_{2}\right), 84.1 \quad\left(p-\mathrm{CH}_{3} \underline{C}_{6} \mathrm{H}_{4} \mathrm{CH}\left(\mathrm{CH}_{3}\right)_{2}\right)$, $83.5 \quad\left(p-\mathrm{CH}_{3} \underline{C}_{6} \mathrm{H}_{4} \mathrm{CH}\left(\mathrm{CH}_{3}\right)_{2}\right), \quad 82.3 \quad(p-$ $\left.\mathrm{CH}_{3} \underline{C}_{6} \mathrm{H}_{4} \mathrm{CH}\left(\mathrm{CH}_{3}\right)_{2}\right), 55.2\left(\mathrm{CO}^{2} \mathrm{H}_{2}\right), 53.5\left(\underline{\mathrm{C}} \mathrm{H}_{2} \mathrm{NH}\right)$, $51.6\left(\mathrm{CH}_{2} \mathrm{Ph}\right), 31.1\left(p-\mathrm{CH}_{3} \mathrm{C}_{6} \mathrm{H}_{4} \underline{\mathrm{C}} \mathrm{H}\left(\mathrm{CH}_{3}\right)_{2}\right), 24.4(p-$ $\left.\mathrm{CH}_{3} \mathrm{C}_{6} \mathrm{H}_{4} \mathrm{CH}\left(\underline{C H}_{3}\right)_{2}\right), 20.1 \quad\left(p-\mathrm{CH}_{3} \mathrm{C}_{6} \mathrm{H}_{4} \mathrm{CH}\left(\underline{\mathrm{C}} \mathrm{H}_{3}\right)_{2}\right)$, $18.5\left(p-\mathrm{CH}_{3} \mathrm{C}_{6} \mathrm{H}_{4} \mathrm{CH}\left(\mathrm{CH}_{3}\right)_{2}\right)$. Anal. calcd. for $\mathrm{C}_{29} \mathrm{H}_{32} \mathrm{RuN}_{3} \mathrm{OCl} \mathrm{C}, 60.56 ; \mathrm{H}, 5.61 ; \mathrm{N}, 7.31$. Found: $\mathrm{C}$, $60.35 ; \mathrm{H}, 5.85 ; \mathrm{N}, 7.60$. 


\section{Computational methods}

Density functional theory calculations were performed on the ruthenium (1-3)c complexes using GAUSSIAN $03^{19}$ suit of quantum chemical programs. The Becke three parameter exchange functional in conjunction with Lee-Yang-Parr correlation functional (B3LYP) has been employed in the study. ${ }^{20}$ The SDD valence basis set was used for ruthenium atom, ${ }^{21}$ while all other atoms are treated at the $6-31 \mathrm{G}(\mathrm{d})$ basis set. ${ }^{22}$ All stationary points are characterized as minima by evaluating Hessian indices on the respective potential energy surfaces. A tight SCF convergence criteria $\left(10^{-8}\right.$ a.u.) was used for all calculations. Natural Bond Orbital (NBO) analysis was performed using the NBO $3.1^{23}$ program implemented in the GAUSSIAN 03 package.

Inspection of the metal-ligand donor-acceptor interactions was carried out using the Charge Decomposition Analysis $(\mathrm{CDA})^{24}$ which is a valuable tool in analysing the interactions between molecular fragments on a quantitative basis, with an emphasis on the electron donation. ${ }^{25}$ The orbital contributions in the ruthenium (1-3)c complexes can be divided into three parts:

(i) $\sigma$-donation from the $[\mathrm{NHC}]^{-} \rightarrow[\mathrm{Ru}(p$-cymene $)$ $\mathrm{Cl}]^{+}$fragments, (ii) $\pi$-back donation from $[\mathrm{NHC}]^{-} \leftarrow[\mathrm{Ru}(p$ cymene)Cl] ${ }^{+}$fragments and

(iii) repulsive polarization $(r)$.

The CDA calculations were performed using the program AOMix ${ }^{26}$ using the B3LYP/SDD, 6-31G(d) wave function. Molecular orbital (MO) compositions and the overlap populations were calculated using the AOMix program. The analysis of the MO compositions in terms of occupied and unoccupied fragment orbitals (OFOs and UFOs respectively), construction of orbital interaction diagrams, the CDA were performed using the AOMix-CDA. ${ }^{27}$

\section{Results and discussion}

A series of ruthenium (1-3)c complexes was stabilized over amido-functionalized $\mathrm{N}$-heterocyclic carbene ligands namely, [1-(R)-3- $N$-(benzylacetamido)imidazol2-ylidene] $\left[\mathrm{R}=\mathrm{Me}, i\right.$-Pr and $\left.\mathrm{CH}_{2} \mathrm{Ph}\right]$. Specifically, the ruthenium (1-3)c complexes were synthesized by a commonly used carbene transfer route from their respective silver (1-3)b complexes by treatment with $\left[\mathrm{Ru}(p \text {-cymene }) \mathrm{Cl}_{2}\right]_{2}$ in $65-76 \%$ yield (scheme 1$)$.

As expected, the ${ }^{13} \mathrm{C}\left\{{ }^{1} \mathrm{H}\right\}$ NMR spectrum of the (1-3)c complexes showed the appearance of a slightly

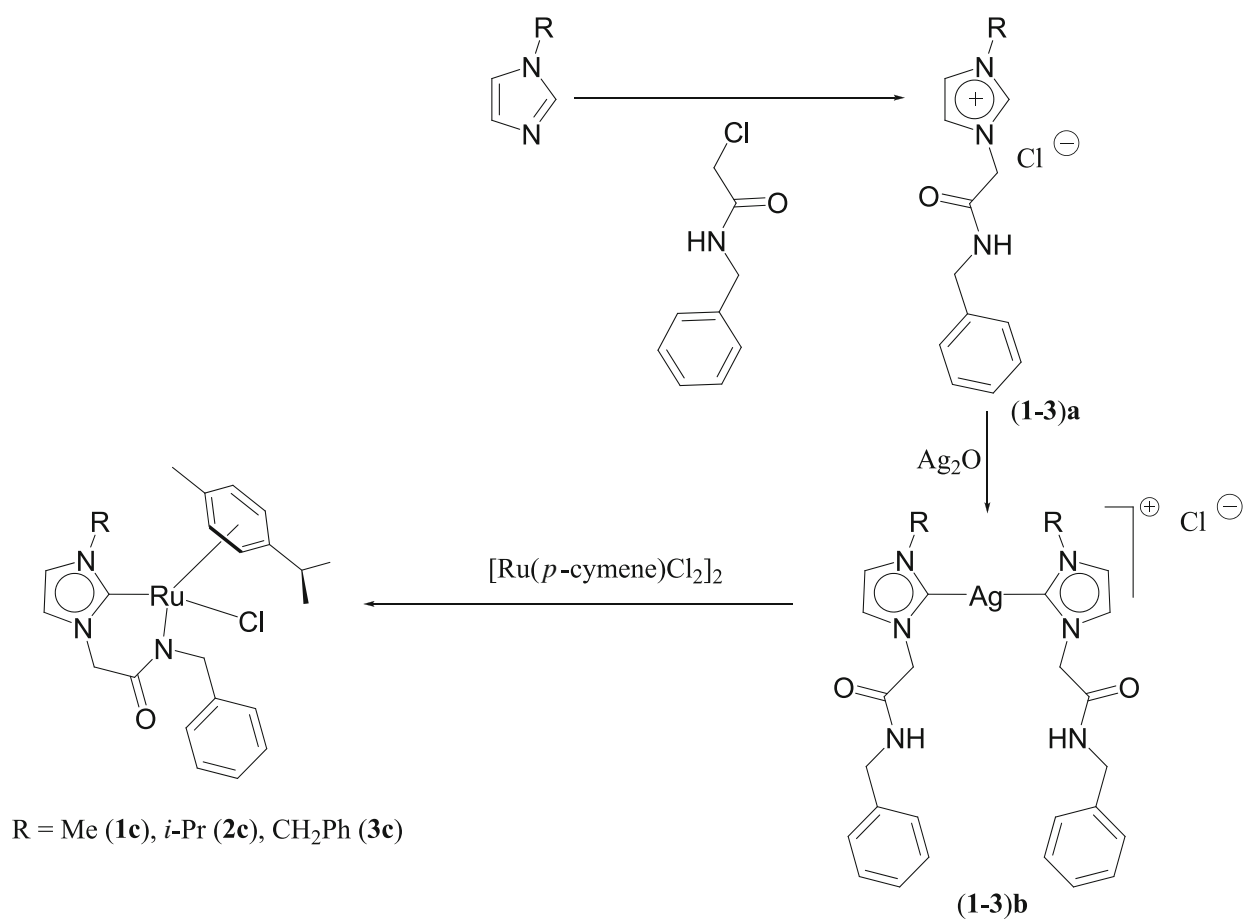

Scheme 1. Synthesis of Ru-NHC (1-3)c complexes 
upfield shifted $(\mathrm{N} C \mathrm{~N}-\mathrm{Ru})$ resonance at $c a .175 .2-$ $175.9 \mathrm{ppm}$. In the ${ }^{1} \mathrm{H}$ NMR spectrum, the amido $(\mathrm{CON} \underline{H})$ resonance was conspicuous by its absence, unlike in the silver (1-3)b complexes, for which it appeared at $c a$. 9.12-9.36 ppm and thus suggestive of ligand chelation to the metal center after deprotonation of the amido sidearm moiety. It is worth noting that the deprotonation of the amido-sidearm resulting in the chelation to the ruthenium center occurred in the absence of any additional base. Interestingly, the $\left(\mathrm{C} \mathrm{H}_{2}\right)$ moieties in the (1-3)c complexes were found to be diastereotopic in nature as it appeared as two set of doublets exhibiting two-bond geminal coupling $\left({ }^{2} \mathrm{~J}_{\mathrm{HH}}\right)$ of $14-16 \mathrm{~Hz}$ in the ${ }^{1} \mathrm{H}$ NMR spectra.

The molecular structures of the ruthenium (1-3)c complexes showed chelation of the N-heterocyclic carbene through an $\mathrm{N}$-amido functionalized sidearm and a carbene moiety to the metal center (figure 2 and see Supporting Information figures $\mathrm{S} 1-\mathrm{S} 2$ and table S1). The aryl group of the cymene moiety was seen bound in a $\eta^{6}$-fashion to the metal center in these complexes. The (1-3)c complexes, having chelated amidofunctionalized $\mathrm{N}$-heteroyclic carbene ligands, closely resemble their non-chelated counterparts. ${ }^{28}$

The Ru- $\mathrm{C}_{\text {carbene }}$ bond distances in (1c) [2.0172(19) $\AA]$, (2c) [2.033(5) $\AA$ ] and (3c) [2.019(3) $\mathrm{A}]$, were in close agreement with the sum of individual covalent radii of $\mathrm{Ru}$ and $\mathrm{C}$ atoms $(2.018 \AA)^{29}$ and with other reported ruthenium $\mathrm{N}$-heterocyclic carbene complexes, namely, $[\mathrm{RuCl}\{2-(4,4-$ dimethyl4,5-dihydrooxazol-2-yl)-(3-mesityl)imidazolidene $(p$-cymene $)] \mathrm{PF}_{6} \quad[2.038(3) \AA],{ }^{29} \quad[\operatorname{RuCl}\{2-(4(\mathrm{~S})-t$ butyl-4,5-dihydrooxazol-2-yl)-(3-mesityl)imidazolidene ( $p$-cymene)] $\mathrm{PF}_{6} \quad[2.03(2) \AA],{ }^{29}$ and [ethylene-bis (N-methylimidazolidene) $\left.\mathrm{RuCl}\left(\mathrm{PF}_{6}\right)\right] \quad[2.033(15) \AA] .{ }^{30}$ Additionally, the $\mathrm{Ru}-\mathrm{Cl}$ bond and $\mathrm{Ru}-\mathrm{C}_{\text {centroid }}$ bond distances in (1c) [2.4256(14) $\AA$ and $1.706 \AA$ ], (2c) [2.4325(8) $\AA$ and $1.712 \AA]$ and (3c) [2.4404(7) $\AA$ and $1.719 \AA]$ were comparable to the $[\mathrm{RuCl}$ \{2-(4,4-dimethyl-4,5-dihydrooxazol-2-yl)-(3-mesityl) imidazolidene $\}(p$-cymene) $] \mathrm{PF}_{6}[2.420(1) \AA$ and $1.708 \AA] .{ }^{29}$ Along the same line, the $\mathrm{Ru}-\mathrm{N}_{\text {amido }}$ bond distances in (1c) $[2.125(5) \AA]$, (2c) $[2.126(2) \AA]$ and (3c) [2.1074(16) $\mathrm{A}]$ also match well with the other ruthenium complexes namely, [hydridotris(pyrazolyl)borate] $\mathrm{Ru}\left[\mathrm{P}(\mathrm{OMe})_{3}\right]_{2}(\mathrm{NHPh})[2.1012(15) \AA]^{31}$ and [2-(benzoimidazol-2-yl)-6-(3,5-dimethylpyrazol-1-yl)pyridine \}] $\mathrm{RuCl}\left(\mathrm{PPh}_{3}\right)_{2}[2.088(17) \AA] .{ }^{32}$

The electronic spectra of the complexes (1-3)c in acetonitrile showed a strong absorption at 242$249 \mathrm{~nm}\left(2223-2371 \mathrm{M}^{-1} \mathrm{~cm}^{-1}\right)$ attributed to a ligand based transition along with two shoulders appear at

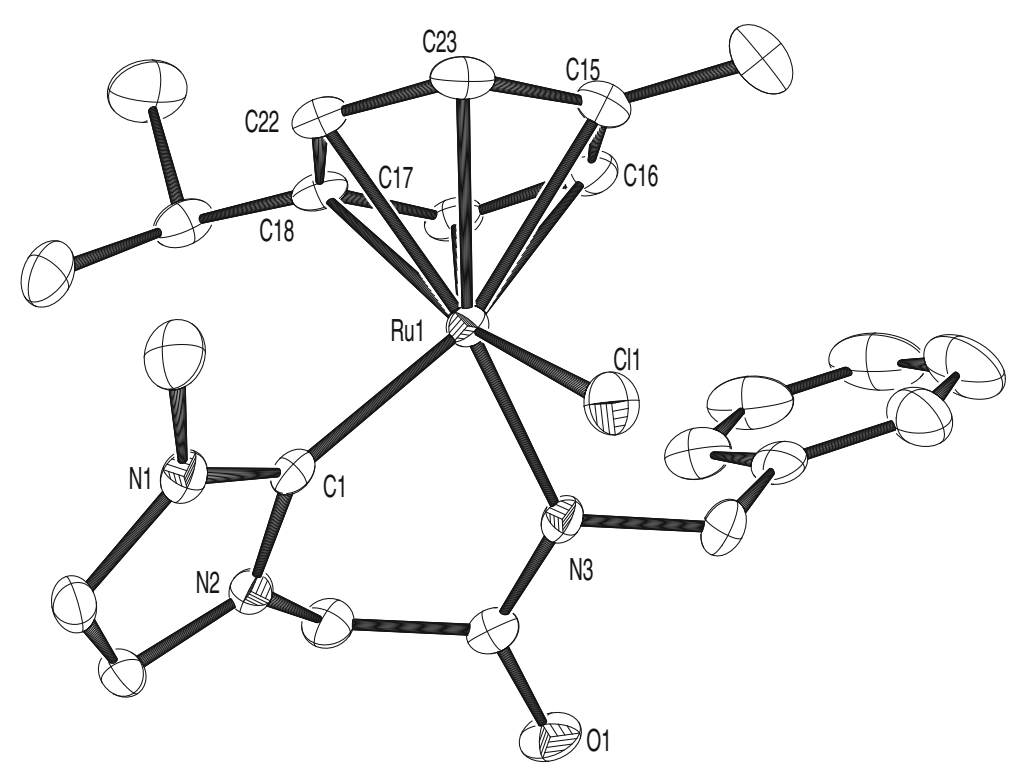

Figure 2. ORTEP diagram of 1c with thermal ellipsoids drawn at $50 \%$ probability level. Selected bond length $(\AA)$ and bond angles $\left({ }^{\circ}\right)$ : Ru1C1 2.0172(19), Ru1-N3 2.1074(16), Ru1-Cl1 2.4404(7), C1-Ru1-N3 84.31(7), C1-Ru1-Cl1 87.31(6), N1-C1-N2 104.02(16). 
$392-395 \mathrm{~nm} \quad\left(314-373 \mathrm{M}^{-1} \mathrm{~cm}^{-1}\right)$ and $459-463 \mathrm{~nm}$ (249-286 $\mathrm{M}^{-1} \mathrm{~cm}^{-1}$ ) (see Supporting Information figures S3-S5 and table S2). Furthermore, the electrochemical behaviour of (1-3)c complexes as analysed by cyclic voltammetry studies showed two quasireversible oxidation responses on the positive side of SCE at $0.93-0.94 \mathrm{~V}$, attributable to a $\mathrm{Ru}(\mathrm{II})-\mathrm{Ru}(\mathrm{III})$ oxidation process, ${ }^{33}$ and at $1.41-1.44 \mathrm{~V}$, attributable to a $\mathrm{Ru}(\mathrm{III})-\mathrm{Ru}(\mathrm{IV})$ oxidation process ${ }^{34}$ (see Supporting Information figures S6-S8 and table S3).

The NHC-Ru interaction in the (1-3)c complexes has been investigated using density functional theory (DFT) studies by computing the minimum energy structure at the B3LYP level of theory using 6-31G(d)/SDD basis set through geometry optimization using atomic coordinates adopted from the X-ray analysis and subsequently undertaking the single point calculation. The carbene-Ru interaction was probed by performing the post-wave function analysis using the Natural Board Orbital (NBO) calculations on the (1-3)c complexes as well as on the corresponding N-heterocyclic carbene ligand and the metal ion fragments (see Supporting Information tables S4-S12).

Both the natural and Mulliken charge analysis showed significant increase of electron density at the metal center in the (1-3)c complexes with subsequent reduction of the same on the free NHC ligand fragment (see Supporting Information tables S4-S6). The RuNHC bond in (1-3)c involved an interaction of the $s p^{2}$ hybridized carbene carbon orbital with a $s d$ hybridized ruthenium orbital as observed from NBO analysis studies (see Supporting Information tables S7-S8).

The Charge Decomposition Analysis (CDA) performed on the (1-3)c complexes provided insight about the relative extent of NHC ligand to metal $\sigma$-donation denoted by $d$ and the metal to NHC ligand $\pi$-back donation denoted by $b$, occurring in these complexes (see Supporting Information table S9). The high $d / b$ ratio observed for the $\mathbf{1 c}(5.63), \mathbf{2 c}(5.96)$ and $\mathbf{3 c}$ (5.28) complexes suggested the predominance of the $\sigma$-interaction in the carbene-Ru bond, and which is in agreement with the as such good $\sigma$-donation ability of the N-heterocyclic carbene ligands. It is worth noting that the $\sigma$-donation $(d)$ and $\pi$-back donation $(b)$ values obtained for (1-3)c represent the donation occurring between a metal center of the $[\mathrm{Ru}(p \text {-cymene }) \mathrm{Cl}]^{+}$ and a functionalized NHC-ligand fragment. This particular interaction is a composite of two interactions namely a $\mathrm{Ru}-\mathrm{C}_{\text {carbene }}$ interaction and a $\mathrm{Ru}-\mathrm{N}_{\text {amido }}$ interaction that arise out of chelation of the NHC ligand to the metal center through an $\mathrm{N}$-amido sidearm and a carbene moiety.

The molecular orbital (MO) correlation diagram, constructed from the individual fragment molecular orbitals (FMOs) of the free NHC ligand and the $[\mathrm{Ru}(p$ cymene)Cl] ${ }^{+}$fragments using AOMix program, ${ }^{35}$ provided useful insights on the nature of the Ru-NHC interaction in the (1-3)c complexes (figure 3 and see

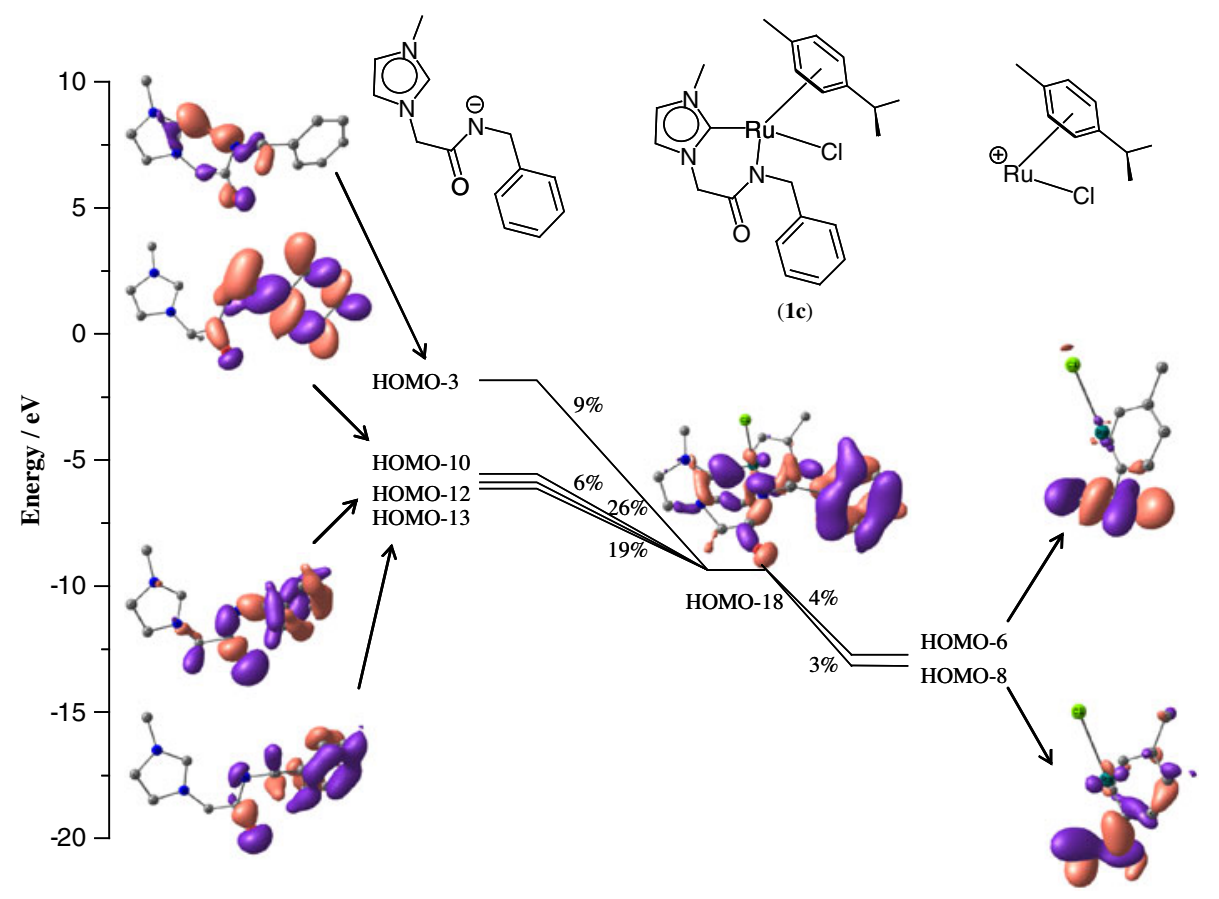

Figure 3. Simplified orbital interaction diagram showing the major contribution of NHC-ruthenium bond in 1c. 
Supporting Information figures S9-S10). Of particular interest are the molecular orbitals representing the $\mathrm{Ru}-\mathrm{NHC} \sigma$-interaction that comprized of the interaction of the carbene lone pair with the ruthenium based orbital namely, 1c (HOMO-18), 2c (HOMO-18) and 3c (HOMO-20). In this regard, it is worth noting that these $\mathrm{Ru}-\mathrm{NHC} \sigma$-bonding molecular orbitals, by virtue of being low lying, remain unaffected by the incoming electrophiles or nucleophiles and thereby contribute toward the stability of the ruthenium (1-3)c complexes.

\section{Conclusion}

In summary, a series of new ruthenium (1-3)c complexes was synthesized employing the transmetallation route from its silver (1-3)b complexes. The molecular structures of the (1-3)c complexes revealed chelation of the $\mathrm{N}$-heterocyclic carbene ligand to the metal center through an $\mathrm{N}$-amido sidearm moiety and a carbene moiety. The density functional theory studies carried out on these ruthenium complexes reflected strong $\sigma$-donating nature of the N-heterocyclic carbene ligands. Further computational studies suggested that the low lying and deeply seated molecular orbitals corresponding to the NHC-Ru $\sigma$-interaction pointed towards a greater stability of the NHC-Ru bond in these complexes.

\section{Electronic supplementary information}

CCDC-676785 (1c), CCDC-697327 (2c) and CCDC685445 (3c) contain the supplementary crystallographic data for this paper. The data can be obtained free of charge at www.ccdc.cam.ac.uk/conts/retrieving.html or from the Cambridge Crystallographic Data Center, 12 Union Road, Cambridge CB2 1EZ, UK; Fax: (internat.) +44-1223/336-033; E-mail: deposit@ccdc.cam.ac.uk. Complete reference [19], CIF files giving X-ray crystallographic data of (1-3)c, UV-vis and CV data, the B3LYP coordinates of the optimized geometries of (1-3)c, computational data and the control experiment data are available free of charge via the internet at http://www.ias.ac.in/chemsci.

\section{Acknowledgements}

We thank Board of Research in Nuclear Sciences (BRNS) Mumbai, for financial support to carry out this research. We are grateful to the National Single Crystal X-ray Diffraction Facility and Sophisticated Analytical Instrument Facility at IIT Bombay, Mumbai, India, for the crystallographic and other characterization data. Computational facilities from the IIT Bombay
Computer Center are gratefully acknowledged. SK and MNR thank Council of Scientific Industrial Research (CSIR), New Delhi, for research fellowship.

\section{References}

1. (a) Boyer J L, Rochford J, Tsai M K, Muckerman J T and Fujita E 2010 Coord. Chem. Rev. 254 309; (b) Keceli E and Ozkar S 2008 J. Mol. Catal. A 286 87; (c) Chatterjee D and Mitra A $2008 \mathrm{~J}$. Mol. Catal. A 282 124; (d) Dragutan V, Dragutan I, Delaude L and Demonceau A 2007 Coord. Chem. Rev. 251 765; (e) Fujita S I, Akihara S, Zhao F, Liu R, Hasegawa M and Arai M 2005 J. Catal. 236101

2. (a) Martinez R, Simon M O, Chevalier R and Pautigny C 2009 J. Am. Chem. Soc. 131 7887; (b) Murahashi S I, Nakae T, Terai H and Komiya N 2008 J. Am. Chem. Soc. 13011005

3. (a) Colacot T J 2011 Platinum Metals Rev. 55 84; (b) Xue L and Lin Z 2010 Chem. Soc. Rev. 39 1692; (c) Denmark S E and Regens C S 2008 Acc. Chem. Res. 41 1486; (d) Miyaura N and Suzuki A 1995 Chem. Rev. 952457

4. (a) Zhu L, Yempally V, Isrow D, Pellechia P J and Captain B 2010 J. Organomet. Chem. 695 1; (b) Kalyani D, Deprez N R, Desai L V and Sanford M S 2005 J. Am. Chem. Soc. 127 7330; (c) Fiedler D, Leung D H, Bergman R G and Raymond K V 2005 Acc. Chem. Res. 38 351; (d) Cundari $\mathrm{T} \mathrm{R}$, Klinckman $\mathrm{T} \mathrm{R}$ and Wolczanski P T 2002 J. Am. Chem. Soc. 1241481

5. (a) Zeng F and Yu Z 2008 Organometallics 27 2898; (b) Ikariya T and Blacker A J 2007 Acc. Chem. Res. 40 1300

6. (a) Vougioukalakis G C and Grubbs R H 2010 Chem. Rev. 110 1746; (b) Colacino E, Martinez J and Lamaty F 2007 Coord. Chem. Rev. 251 726; (c) Clavier H, Grela K, Kirschning A, Mauduit M and Nolan S P 2007 Angew. Chem. Int. Ed. 466786

7. Chatterjee D 2008 Coord. Chem. Rev. 252176

8. (a) Tominaga K I and Sasaki Y 2004 J. Mol. Catal. A 220 159; (b) Pittman C U and Wileman G M $1981 \mathrm{~J}$. Org. Chem. 461901

9. Panizza M and Cerisola G 2009 Chem. Rev. 1096541

10. Boren B C, Narayan S, Rasmussen L K, Zhang L, Zhao H, Lin Z, Jia G and Fokin V V 2008 J. Am. Chem. Soc. 1308923

11. (a) Liddle S T, Edworthy I S and Arnold P L 2007 Chem. Soc. Rev. 36 1732; (b) Mataa J A, Poyatos M and Peris E 2007 Coord. Chem. Rev. 251 841; (c) Kühl O 2007 Chem. Soc. Rev. 36592

12. (a) Ray S, Asthana J, Tanski J M, Shaikh M M, Panda D and Ghosh P 2009 J. Organomet. Chem. 694 2328; (b) Ray S, Mohan R, Singh J K, Samantaray M K, Shaikh M M, Panda D and Ghosh P 2007 J. Am. Chem. Soc. 12915042

13. (a) Samantaray M K, Dash C, Shaikh M M, Pang K, Butcher R J and Ghosh P 2011 Inorg. Chem. 50 1840; (b) Dash C, Shaikh M M, Butcher R J and Ghosh P 2010 Inorg. Chem. 49 4972; (c) Dash C, Shaikh M M, Butcher 
R J and Ghosh P 2010 Dalton Trans. 39 2515; (d) John A, Shaikh M M and Ghosh P 2009 Dalton Trans. 10581; (e) Samantaray M K, Shaikh M M and Ghosh P 2009 J. Organomet. Chem. 694 3477; (f) Dash C, Shaikh M M and Ghosh P 2009 Eur. J. Inorg. Chem. 1608; (g) Ray L, Barman S, Shaikh M M and Ghosh P 2008 Chem. Eur. J. 14 6646; (h) Ray L, Shaikh M M and Ghosh P 2007 Dalton Trans. 4546; (i) Ray L, Katiyar V, Barman S, Raihan M J, Nanavati H, Shaikh M M and Ghosh P 2007 J. Organomet. Chem. 692 4259; (j) Samantaray M K, Katiyar V, Pang K, Nanavati H and Ghosh P 2007 J. Organomet. Chem. 692 1672; (k) Ray L, Shaikh M M and Ghosh P 2007 Organometallics 26 958; (1) Ray L, Katiyar V, Raihan M, Nanavati H, Shaikh M M and Ghosh P 2006 Eur. J. Inorg. Chem. 3724; (m) Samantaray M K, Katiyar V, Roy D, Pang K, Nanavati H, Stephen R, Sunoj R B and Ghosh P 2006 Eur. J. Inorg. Chem. 2975

14. (a) John A and Ghosh P 2010 Dalton Trans. 397183 ; (b) Dash C, Shaikh M M and Ghosh P 2011 J. Chem. Sci. 123 97; (c) Samantaray M K, Pang K, Shaikh M M and Ghosh P 2008 Dalton Trans. 4893; (d) Samantaray M K, Pang K, Shaikh M M and Ghosh P 2008 Inorg. Chem. 47 4153; (e) Ray L, Shaikh M M and Ghosh P 2008 Inorg. Chem. 47 230; (f) Samantaray M K, Roy D, Patra A, Stephen R, Saikh M, Sunoj R B and Ghosh P 2006 J. Organomet. Chem. 6913797

15. Kumar S, Narayanan A, Rao M N, Shaikh M M and Ghosh P 2011 J. Organomet. Chem doi: 10.1016/j.jorganchem.2011.09.007

16. Kumar S, Shaikh M M and Ghosh P 2009 J. Organomet. Chem. 6944162

17. Bennett M A and Smith A K 1974 J. Chem. Soc. Dalton Trans. 233

18. (a) Sheldrick G M 1997 SHELXL-97, Program for refinement of crystal structures (Germany: University of Gottingen); (b) Sheldrick G M 1997 SHELXS-97, Structure solving program (Germany: University of Gottingen)

19. Frisch M J et al. 2004 GAUSSIAN 03: Gaussian 03, Revision C.02, Gaussian, Inc. (Wallingford, CT)
20. (a) Becke A D 1988 Phys. Rev. A 38 3098; (b) Lee C, Yang W and Parr R G 1988 Phys. Rev. B 37785

21. (a) Lu X, Wei S, Wu C M L, Guo W and Zhao L 2011 J. Organomet. Chem. 696 1632; (b) Buntem R, Punyain K, Tantirungrotechai Y, Raithby P R and Lewis J 2010 Bull. Kor. Chem. Soc. 31 934; (c) Wang C and Wu Y D 2008 Organometallics 27 6152; (d) Gomez J, Gemel C, Slugovc C, Wozniak E, Schmid R and Kirchner K 2001 Monatsh. Chem. 1321137

22. Hehre W J, Ditchfield R and Pople J A 1972 J. Chem. Phys. 562257

23. Reed A E, Curtiss L A and Weinhold F 1988 Chem. Rev. $\mathbf{8 8} 899$

24. Dapprich S and Frenking G 1995 J. Phys. Chem. 99 9352

25. (a) Vyboishchikov S F and Frenking G 1998 Chem. Eur. J. 4 1439; (b) Frenking G and Pidun U 1997 J. Chem. Soc., Dalton Trans. 1653

26. Gorelsky S I and Lever A B P 2001 J. Organomet. Chem. 635187

27. Gorelsky S I, Ghosh S and Solomon E I 2006 J. Am. Chem. Soc. 128278

28. Prades A, Viciano M, Sanaú M and Peris E 2008 Organometallics 274254

29. Pauling L 1960 The Nature of The Chemical Bond, 3rd ed. (Cornell University Press: Ithaca, NY) p 224, 256

30. Poyatos M, Maisse-François A, Bellemin-Laponnaz S, Peris E and Gade L H 2006 J. Organomet. Chem. 691 2713

31. Poyatos M, Mas-Marzá E, Sanaù M and Peris E 2004 Inorg. Chem. 431793

32. Conner D, Jayaprakash K N, Gunnoe T B and Boyle P D 2002 Inorg. Chem. 413042

33. Zeng F and Yu Z 2009 Organometallics 281855

34. Gandolfi C, Heckenroth M, Neels A, Laurenczy G and Albrecht M 2009 Organometallics 285112

35. Basuli F; Das A K; Mostafa G; Peng S-M; Battacharya S 2000 Polyhedron 191663

36. Gorelsky S I 1997 AOMix: Program for molecular orbital analysis (Toronto, Canada: York University); http://www.sg-chem.net/ (accessed 30 June 2011) 